\title{
Efficacy of Pre-Treatment with Remimazolam on Prevention of Propofol-Induced Injection Pain in Patients Undergoing Abortion or Curettage: A Prospective, Double-Blinded, Randomized and Placebo-Controlled Clinical Trial
}

\author{
Xuehai Guan (1D ${ }^{1, *}$ \\ Ziyin Jiao ${ }^{1, *}$ \\ Xiaofang Gong' \\ Huiyu Cao' \\ Susu Liu' \\ Hongmeng Lan' \\ Xiaofang Huang' \\ Yanmeng Tan' \\ Bing $X u^{2}$ \\ Chengxin Lin ${ }^{\prime}$ \\ 'Department of Anesthesiology, The \\ First Affiliated Hospital of Guangxi \\ Medical University, Nanning, Guangxi, \\ People's Republic of China; ${ }^{2}$ Department \\ of Rehabilitation, The People's Hospital \\ of Guangxi Zhuang Autonomous Region, \\ Nanning, Guangxi, People's Republic of \\ China
}

*These authors contributed equally to this work

\begin{abstract}
Background: Propofol-induced injection pain (PIP) is a well-known problem in general anesthesia. We hypothesized that pre-treatment with remimazolam prevents PIP in patients undergoing abortion or curettage.

Materials and Methods: In this prospective, single-center, double-blinded, randomized, placebo-controlled clinical trial, adult patients aged 18 to 60 undergoing abortion or curettage were randomly assigned to three groups. Group Lido received system lidocaine (a bolus of $0.5 \mathrm{mg} \mathrm{kg}^{-1}$, iv). Group Remi received remimazolam (a bolus of $0.1 \mathrm{mg} \mathrm{kg}^{-1}$, iv). Group NS received identical volumes of $0.9 \%$ normal saline. Sixty seconds after the injection of lidocaine, remimazolam or saline, patients were injected with propofol at a rate of $12 \mathrm{~mL} / \mathrm{min}$ until the loss of consciousness. The primary outcome was the incidence of PIP at the time of induction using 4-point scale. Secondary outcomes included propofol-induced injection pain, vital signs, the characteristics of anesthesia and surgery, and adverse events.

Results: The incidence of patients with PIP was higher in group NS than that in group Lido and group Remi $(75.7,44.3$, and 42.9\%, respectively, $p<0.001)$. The percentages of patients with moderate PIP were higher in group NS than that in group Lido and group Remi (20.0, 2.9 , and $1.4 \%$, respectively, $p<0.001$ ). Moreover, the consumption of propofol and the incidence of adverse event (hypoxemia and chin lifting) in group Remi were lower than that in group NS and Lido, and less patients got physical movement and cough in group Remi. The recovery time in group NS was longer than that in group Lido and Remi.

Conclusion: Our findings indicate that pre-treatment with remimazolam reduced the incidence and intensity of PIP in abortion or curettage patients, equivalent to that of lidocaine without severe adverse effects.
\end{abstract}

Trial Registration: Chinese Clinical Trial Registry (identifier: ChiCTR2100041805).

Keywords: lidocaine, remimazolam, propofol injection pain, abortion, curettage

\section{Introduction}

Due to its rapid onset and recovery, propofol is popularly used during general anesthesia. However, the intravenous injection of propofol induces local pain and discomforts. The incidence of propofol-induced injection pain (PIP) varies from approximately 28 to $90 \% .^{1-3}$ To reduce the incidence of PIP, many techniques 
have been developed, including pre-treatment or mixed use with medium-chain and long-chain triglycerides, ${ }^{4}$ pre-treatment or mixed use with lidocaine, ${ }^{5-9}$ nonsteroidal anti-inflammatory drugs, ${ }^{10}$ magnesium sulfate, ${ }^{6,11}$ dexmedetomidine, ${ }^{12}$ opioids, ${ }^{13-16}$ or ketamine. $^{11,17}$ Although these strategies relieved PIP in varying degrees, the adverse event of these drugs such as emergence agitation, ${ }^{18}$ laryngospasm, ${ }^{19}$ pulmonary embolism, ${ }^{20}$ gastrointestinal ulcer, ${ }^{10}$ lengthy onset ${ }^{21}$ or tinnitus and dizziness ${ }^{22}$ limit their widespread clinical use. More patients complained of tinnitus or dizziness after the injection of lidocaine, ${ }^{22}$ and the addition of lidocaine may disrupt the stability of propofol emulsions and may cause pulmonary embolism. ${ }^{19}$ These drawbacks limited the use of lidocaine for preventing PIP. Consequently, there is a need for finding news drugs to decrease the incidence of PIP.

Remimazolam is an ultrashort-acting benzodiazepine, acting on GABA receptors to induce sedation. It is developed for procedural sedation. ${ }^{23-25}$ Unlike midazolam, remimazolam differs from all other benzodiazepines by its carboxylic ester linkage, metabolized by tissue esterase rapidly to inactive metabolites only. ${ }^{23-25}$ Remimazolam provided adequate procedural sedation for endoscopy, and faster recovery than midazolam. ${ }^{26-29}$ Data on the influence of remimazolam on PIP during abortion or curettage have not been published. Therefore, we designed this prospective, single-center, double-blinded, randomized, placebo-controlled clinical trial to investigate the efficacy of remimazolam $0.1 \mathrm{mg} / \mathrm{kg}$ and lidocaine $0.5 \mathrm{mg} /$ $\mathrm{kg}$ compared to placebo in the prevention of PIP during abortion or curettage.

\section{Materials and Methods}

\section{Patients}

The randomization schedule was computer-generated by using Epical 2000 soft. According to the randomization schedule, all 210 patients were randomly divided into three groups $(\mathrm{n}=70$ in each group) in a 1:1:1 group allocation to receive either lidocaine (Group Lido, received system lidocaine, a bolus of $0.5 \mathrm{mg} \mathrm{kg-1,} \mathrm{iv),}$ remimazolam (Group Remi, received remimazolam,

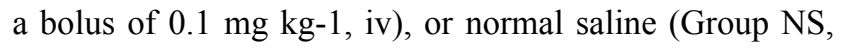
received equivalent volume of $0.9 \%$ normal saline). Sealed envelopes were used for concealment of study group allocation until the pretreatment drug was prepared. An assistor who did not participate in anesthesia induction prepared all drugs. Both patients and investigators were blinded to the randomized grouping allocation and the drugs.

\section{Study Protocol}

After obtaining approval from the Ethics Committee of the first affiliated hospital of Guangxi Medical University and written consent from the selected patients, we enrolled two hundred ten ASA physical status 1 and 2, aged 18-60 years, who were scheduled for elective abortion or curettage procedures with general anesthesia. Patients with liver and kidney dysfunction, drug allergy, nervous system or cardiovascular disease, obesity, difficult airway were excluded. Patients receiving analgesics were also excluded. The study was registered in the Chinese Clinical Trial Registry (ChiCTR2100041805, Principal investigator: Xuehai Guan, Date of registration: 2021-1-6). This study was conducted in accordance with the Declaration of Helsinki. All participants were informed about the purpose of the trial.

Patients were fasted for 6 hours. Only clear liquids were allowed up to 2 hours before the induction of anesthesia. No sedative premedication was given before induction. A 22-gauge cannula was inserted into the vein on the dorsum of the left hand without local anesthetics, at least $10 \mathrm{~min}$ before the induction of anesthesia, and an infusion of Ringer's Lactate $(2 \mathrm{~mL} / \mathrm{kg} / \mathrm{h})$ was started to maintain its patency. The infusion of Ringer's lactate was closed during the induction period.

After entering the operation room, patients were given routine nasal catheter oxygen inhalation of $2 \mathrm{~L} / \mathrm{min}$, and the noninvasive blood pressure, electrocardiogram, and peripheral capillary oxygen saturation $\left(\mathrm{SpO}_{2}\right)$ were monitored. The pre-treating drugs were prepared in a $10-\mathrm{mL}$ syringe with either $10 \mathrm{~mL}$ of normal saline, $0.5 \mathrm{mg} / \mathrm{kg}$ of lidocaine (Shanghai Harvest Pharmaceutical CO., China), or $0.1 \mathrm{mg} / \mathrm{kg}$ of remimazolam (Jiangsu Hengrui Medicine Co., China; diluted with normal saline to $10 \mathrm{~mL}$ ) according to the group allocation by an assistor who did not participate in anesthesia induction. Both patients and investigators were blinded to the randomized grouping allocation and the drugs. All drugs were prepared and stored at room temperature and used within 10 mins. All patients were injected with a mixture of fentanyl (Yichang Humanwell Pharmaceutical Co., China) and atropine (1 ug/kg and $5 \mathrm{ug} / \mathrm{kg}$, respectively). Fifteen seconds later, patients in Group Lido received system lidocaine (a bolus of $0.5 \mathrm{mg} \mathrm{kg}-1$, iv), in Group Remi received remimazolam (a bolus of $0.1 \mathrm{mg} \mathrm{kg}-1$, iv), and 
in Group NS received equivalent volume of $0.9 \%$ normal saline. Sixty seconds after the injection of lidocaine, remimazolam or saline, all patients received propofol (Guangdong JiaBo Pharmaceutical Co., China) at a rate of $12 \mathrm{~mL} / \mathrm{min}$ until loss of consciousness. The sedation was monitored by using the Modified Observer's Assessment Alertness/Sedation Scale (OAA/S; 5: responds readily to their name spoken in a normal tone, 4: lethargic response to their name spoken in a normal tone, 3: response only after their name is called loudly and/or repeatedly; 2: response only after name spoken with mild prodding or shaking; 1: unresponsive to mild prodding or shaking; 0 : unresponsive to noxious stimuli) ${ }^{30}$ with $1 \mathrm{~min}$ interval. If an $\mathrm{OAA} / \mathrm{S}$ score of 0 was not achieved, infusions of propofol continued until it reached 0 .

\section{Measurements}

The primary outcome of this study was the incidence of PIP. Secondary outcomes included the intensity of PIP, vital signs, and adverse events, including hypotension, bradycardia ( $<50$ beats/min), hypoxemia $\left(\mathrm{SpO}_{2}<90 \%\right)$, chin lifting, physical movement and cough. Investigators who were blinded to the groups location evaluated the severity of propofol injection pain according to the 4-point pain scale every 5 seconds during anesthesia induction: grade 0 , no pain; grade 1 , mild pain but no physical movement; grade 2, moderate pain, pain accompanied by physical activity when the anesthetist asked, or during the injection; and grade 3 , severe pain, accompanied by facial pain, painful expression, or strong vocal response, arms retracted, or tears. ${ }^{31}$ The characteristics of anesthesia and surgery, and adverse events were recorded too.

\section{Sample Size}

Our preliminary study revealed that the incidence of PIP was about $50 \%$ in our department which was among the previous study between 28 and 90\%. We hypothesized a $50 \%$ reduction in the incidence of pain after propofol administration based on an alpha of 0.05 and a power of $80 \%$. Under these assumptions, 57 patients were included in each group to detect a significant difference. Considering potential loss $(20 \%)$ to follow-up, we increased the sample size to 70 in each group.

\section{Statistical Methods}

All data are expressed as numbers $(\%)$ or the mean \pm SD. Continuous data of patients among the three groups were compared by one-way analysis of variance or two-way analysis of variance where appropriate. Categorical data were compared by $x^{2}$ test or Fisher's test, as appropriate. All statistical analyses were performed with IBM SPSS 25.0 statistical software. A $p$-values or corrected $p$-values of 0.05 were defined as statistically significant.

\section{Results}

All 210 patients enrolled in the study were evaluable (Figure 1; from 2021-1-6 to 2021-5-6). There were no significant differences among the three groups in demographics - age, height, weight, ASA score rate, or Mallampati score rate (Table 1).

The overall incidence and intensity of PIP during propofol injection in the three groups were showed in Table 2. The incidence of PIP was significantly less in group Lido $(44.3 \%)$ and Remi (42.9\%) than those in group NS $(75.7 \%)(p<0.001)$. There was no significant difference between group Lido and group Remi in incidence of PIP $(p>0.05)$. No significant difference was found in the percentages with mild pain among the three groups. The percentage of patients with moderate pain was significantly less in group Lido (2.9\%) and group Remi (1.4\%) than those in group NS $(20.0 \%)(p<0.001)$. No significant difference was found in the percentages of patients with moderate pain between group Lido and group Remi. Although there was no significant difference among the three groups in the percentages of patients suffering from severe pain $(p>0.05), 2$ cases and 1 case were found in group NS and group Lido, respectively.

The incidence of adverse event is shown in Table 3. There were no differences in the proportion of patients developed hypotension and bradycardia among the three groups. The proportion of patients with hypoxemia in group Remi (11.4\%) was lower than that in group NS $(34.3 \%)$ and group Lido $(38.6 \%)(p<0.001)$. The proportion of patients needing chin lift in group Remi (4.3\%) was lower than that in group NS (34.3\%) and group Lido $(38.6 \%)(p<0.001)$. Although there was no significant difference among the three groups in the percentages of patients with physical movement $(p>0.05), 5$ cases, 7 case and 1 case were found in group NS, group Lido and group Remi respectively. Although there was no significant difference among the three groups in the percentages 


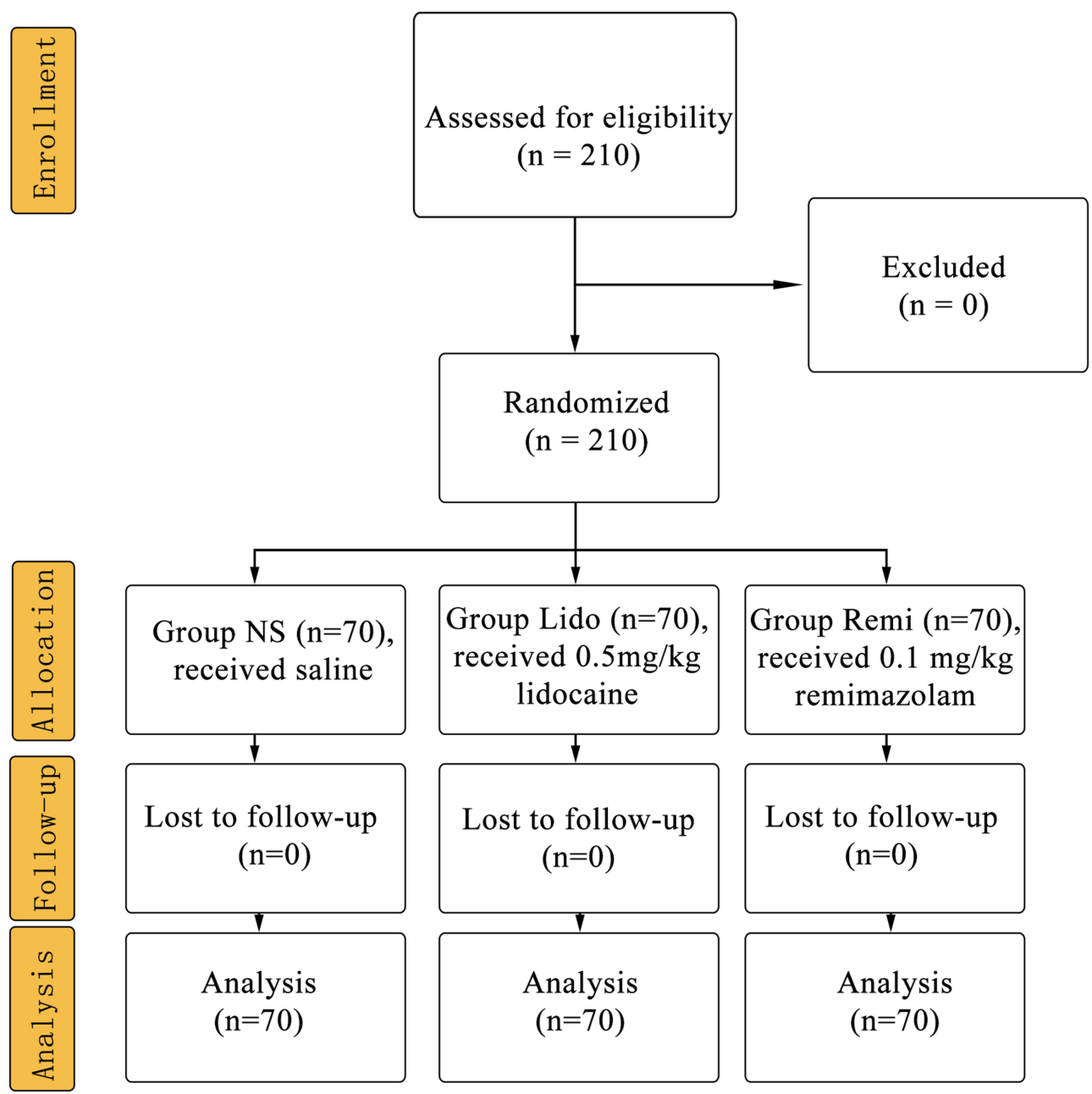

Figure I CONSORT flow of clinical procedures.

Abbreviations: NS, normal saline; Lido, lidocaine; Remi, remimazolam.

of patients with cough $(p>0.05), 1$ case and 4 cases were found in group NS and group Lido respectively.

The characteristic of anesthesia and surgery is shown in Table 4. The overall consumption of propofol in group Remi $(119.4 \pm 25.33 \mathrm{mg})$ was lower than those in group NS $(134.6 \pm 28.37 \mathrm{mg})$ and group Lido $(140.1 \pm 37.16 \mathrm{mg})$ $(p<0.001)$. There was no difference in the length of anesthesia and surgery among three groups. The recovery time was shorter in group Lido $(2.20 \pm 1.73 \mathrm{~min})$ and group Remi $(2.09 \pm 3.08 \mathrm{~min})$ than that in group NS $(3.74 \pm 1.81 \mathrm{~min})(p<0.001)$. But there was no difference in recovery time between group Lido and group Remi.

There were no differences in the systolic blood pressure, diastolic blood pressure, mean blood pressure, heart rate and $\mathrm{SpO}_{2}$ at any time point among the three groups (Figure 2).

Table I Demographic Data of the Patients ( $\mathrm{n}=70$ in Each Group)

\begin{tabular}{|l|l|l|l|l|}
\hline Parameter & Group NS & Group Lido & Group Remi & p value \\
\hline Age $(\mathrm{yrs})$ & $30.73 \pm 6.83$ & $32.01 \pm 7.66$ & $29.90 \pm 5.72$ & 0.1848 \\
Height $(\mathrm{cm})$ & $159.0 \pm 5.19$ & $157.5 \pm 12.66$ & $158.9 \pm 5.30$ & 0.4918 \\
Body weight (kg) & $54.14 \pm 10.44$ & $54.09 \pm 7.81$ & $53.45 \pm 7.84$ & 0.8830 \\
ASA score (I/II) & $63 / 7$ & $60 / 10$ & $62 / 8$ & 0.7277 \\
Mallampati (I/II) & $14 / 56$ & $16 / 54$ & $17 / 53$ & 0.8254 \\
\hline
\end{tabular}

Notes: Data are displayed as means \pm SD or numbers of cases. No statistically significant differences between groups were noted. Abbreviations: NS, normal saline; Lido, lidocaine; Remi, remimazolam. 
Table 2 Incidence of Propofol Induced Injection Pain ( $=70$ in Each Group)

\begin{tabular}{|c|l|l|l|}
\hline Group & NS & Lido & Remi \\
\hline Patients with pain [No. (\%)] & $53(75.7 \%)$ & $31(44.3 \%) * * *$ & $30(42.9 \%) * * *$ \\
\hline Severity of pain [No. (\%)] & & & \\
0 & $17(24.3 \%)$ & $39(55.7 \%) * * *$ & $40(57.1 \%) * * *$ \\
1 & $37(52.9 \%)$ & $28(40.0 \%)$ & $29(41.4 \%)$ \\
2 & $14(20.0 \%)$ & $2(2.9 \%) * * *$ & $1(1.4 \%) * * *$ \\
3 & $2(2.9 \%)$ & $1(1.4 \%)$ & $0(0 \%)$ \\
\hline
\end{tabular}

Notes: Data are displayed as numbers of cases. Chi-squared tests was used to analyze the incidence of PIP. *** $p<0.001$, compared with group NS.

Abbreviations: NS, normal saline; Lido, lidocaine; Remi, remimazolam.

Table 3 Incidence of Adverse Event Between Groups ( $n=70$ in Each Group)

\begin{tabular}{|l|c|c|c|}
\hline Group & NS & Lido & Remi \\
\hline Hypotension & 2 & 5 & 4 \\
Bradycardia $(<50$ beats/ min) & 0 & 0 & 0 \\
Hypoxemia $\left(\mathrm{SpO}_{2}<90 \%\right)$ & 24 & 27 & $8^{\text {***, }}$ \\
Chin lift & 24 & 27 & $3^{\text {*** }}$ \\
Physical movement & 5 & 7 & 1 \\
Cough & 1 & 4 & 0 \\
\hline
\end{tabular}

Notes: Data are displayed as numbers of cases. Chi-squared tests was used to analyze the incidence of adverse event. $* * * p<0.001$, compared with group NS; \#\# $p$ < 0.00I, compared with group Lido.

Abbreviations: NS, normal saline; Lido, lidocaine; Remi, remimazolam.

\section{Discussion}

To the best of our knowledge, this was the first findings revealed that pre-treatment with remimazolam $(0.1 \mathrm{mg} / \mathrm{kg}$, iv) effectively reduced the incidence and intensity of PIP in abortion or curettage patients, equivalent to that of lidocaine without severe adverse effects. Moreover, pretreatment with remimazolam could reduce the consumption of propofol and the incidence of adverse event, and shorter the recovery time.

Propofol has become a popular sedative agent. Due to its rapid onset and recovery, propofol was widely used in endoscopy, abortion and curettage. However, PIP is a common adverse event. ${ }^{32,33}$ The incidence of PIP varies from approximately 28 to $90 \%{ }^{1-3}$ But the definite pathophysiological mechanism of PIP is still unknown. Many factors are related to PIP, such as concentration of free propofol in aqueous solution, type of preparation, oil and solvent, injection technology (injection site, ${ }^{34}$ injection speed, ${ }^{35}$ intravenous infusion, puncture technology, syringe material), blood buffering, filtration treatment, age, sex, and so on. To reduce the incidence of PIP, many techniques have been developed, including medium-chain and long-chain triglycerides, pre-treatment or mixed use with lidocaine, nonsteroidal anti-inflammatory drugs, magnesium sulfate, dexmedetomidine, opioids, or ketamine. These various strategies relieved PIP in varying degrees. Lidocaine is a common local anesthetic, reversibly blocking peripheral pathway. Premedication with lidocaine $0.5 \mathrm{mg} / \mathrm{kg}$ before the injection of propofol reduced the incidence of PIP significantly, which was consistent with previous study. ${ }^{21,36} \mathrm{~A}$ dosage of $40 \mathrm{mg}$ lidocaine is an appropriate dosage to alleviate PIP within the same vein through a local anesthetic effect. Lidocaine reduces PIP through a central analgesic effect and a local anesthetic effect when the dosage reaches $1.5 \mathrm{mg} / \mathrm{kg}^{22}$ In our study, we believe that lidocaine prevented PIP through a local anesthetic effect under the use of $0.5 \mathrm{mg} / \mathrm{kg}$.

The incidence and intensity of PIP was significantly less in group Lido and group Remi than those in group NS. $\gamma$ aminobutyric acid (GABA), a central inhibitory neurotransmitter acting on GABAA receptor and benzodiazepines can enhance the synaptic inhibitory effect of GABAergic neurotransmission. A lot of research showed that benzodiazepine has analgesic effect. For example, intravenous midazolam-a classic benzodiazepine drug administered in conscious sedation doses was found to significantly reduce the affective and motivational component of the pain experience. ${ }^{37}$ Midazolam intervention was revealed to substantially reduce the pain scores and analgesic consumption after knee arthroscopy. ${ }^{38}$ Midazolam was effective in decreasing pain

Table 4 Characteristics of Anesthesia and Surgery ( $n=70$ in Each Group)

\begin{tabular}{|l|l|l|l|l|}
\hline Parameter & Group NS & Group Lido & Group Remi & p value \\
\hline Propofol dose (mg) & $134.6 \pm 28.37$ & $140.1 \pm 37.16$ & $119.4 \pm 25.33 *, \ldots \#$ & 0.0003 \\
Anesthesia time (min) & $10.96 \pm 2.81$ & $9.87 \pm 3.21$ & $10.21 \pm 2.88$ & 0.0723 \\
Surgery time (min) & $6.87 \pm 2.05$ & $8.26 \pm 2.29$ & $7.68 \pm 2.56 *$, & 0.0400 \\
Recovery time (min) & $3.74 \pm 1.81$ & $2.20 \pm 1.73^{* * *}$ & $2.086 \pm 3.08^{* * *}$ & 0.0000 \\
\hline
\end{tabular}

Notes: Data are displayed as means \pm SD. ANOVA was applied to all comparisons, followed by Bonferroni's Multiple Comparison Test. ${ }^{*} p<0.05$, $* * * p<0.00 I$, compared with group NS; $p<0.00$ I, compared with group Lido.

Abbreviations: NS, normal saline; Lido, lidocaine; Remi, remimazolam. 

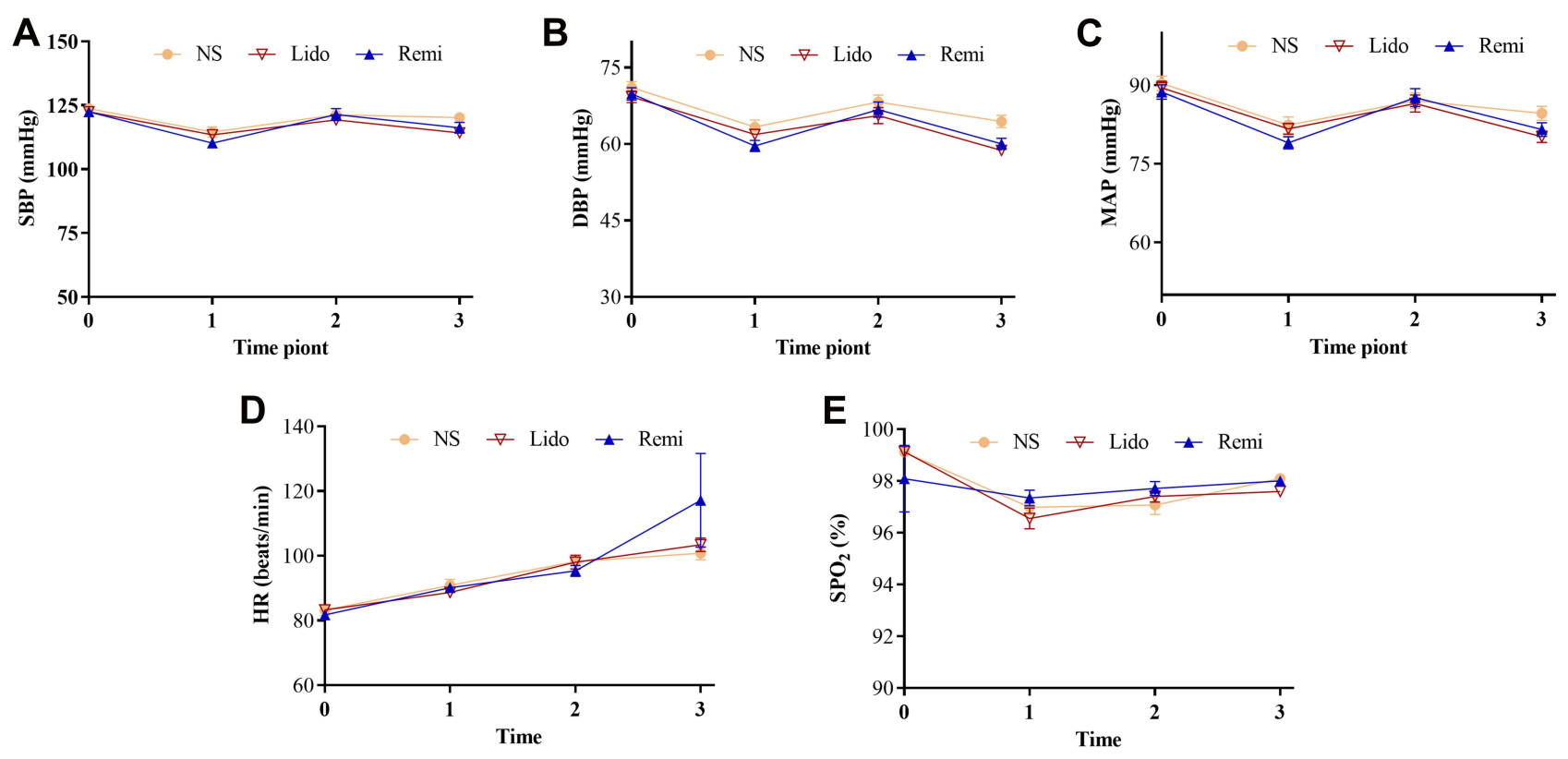

Figure 2 Changes of vital signs (A-E).

Notes: Data are displayed as means \pm SD. Time points: 0 , at the time before the treatment with NS, lidocaine or remimazolam; I, at the beginning of surgery; 2 at the end of surgery; 3 , at the time open eyes.

Abbreviations: NS, normal saline; Lido, lidocaine; Remi, remimazolam; SBP, systolic blood pressure; DBP, diastolic blood pressure; MAP, mean pressure; HR, heart rate; $\mathrm{SPO}_{2}$, peripheral capillary oxygen saturation.

after nasogastric tube insertion. ${ }^{39}$ Remimazolam is an ultrashort-acting benzodiazepine, acting on GABA receptors to induce sedation. ${ }^{23}$ Based on this, we infer that the first underling mechanism remimazolam preventing PIP may be its role by acting on GABA receptors to enhance the synaptic inhibitory effect of GABAergic neurotransmission. Propofol acts on the vein endothelial tissue, and then stimulates the kallikrein kinin system to produce bradykinin, which makes the blood vessel dilate and increase the permeability, and causes the free propofol to contact with the nerve endings on the inner wall of the blood vessel to cause pain. A recent study revealed that treating with remimazolam intraperitoneally alleviated pain behaviors induced by injecting complete Freund's adjuvant in hind paw via regulating bradykinin receptors $\mathrm{B} 1 .^{40}$ Therefore, we deduced that the treatment of remimazolam intravenously alleviated PIP by blocking bradykinin signal too.

The consumption of propofol in group Remi was lower than that in group NS and group Lido. The recovery time in group NS was longer than that in group Lido and group Remi. As mention about, remimazolam can act on GABA receptors to enhance the synaptic inhibitory effect of GABAergic neurotransmission, which may result in less consumption of propofol and faster recovery from sedation in the present study. The incidence of adverse event (hypoxemia, chin lifting, physical movement and cough) in group Remi were lower than that in group NS and group Lido, which was in consistent with previous results that the adverse event was positively correlated with the dosages of propofol. ${ }^{21}$

There were some limitations. First, our study was only conducted in single center. Additionally, we just investigated one dose of remimazolam on the incidence of PIP. In the future, we will coordinate with multi center to evaluate the effect of remimazolam on the prevention of PIP.

In conclusion, our current findings indicate that pretreatment with remimazolam reduced the incidence and intensity of PIP in abortion or curettage patients, equivalent to that of lidocaine without severe adverse effects. Moreover, pre-treatment with remimazolam $0.1 \mathrm{mg} / \mathrm{kg}$ can reduce the consumption of propofol and the incidence of adverse event, and shorter the recovery time.

\section{Data Sharing Statement}

The data generated during the current study are available from the corresponding author (Xuehai Guan) on reasonable request. The study protocol, statistical analysis plan and clinical study report will also be available.

\section{Acknowledgments}

We are grateful to Juanjuan Wang and Xinxin Luo for their kind assistance. This work was supported by the Natural Science Foundation of Guangxi Zhuang Autonomous 
Region (Nos. 2018GXNSFAA281049 and 2015GX NSFBA139133) and the National Natural Science Foundation of China (Nos. 81660200 and 81460176).

\section{Disclosure}

The authors report no conflicts of interest in this work.

\section{References}

1. Wrench IJ, Girling KJ, Hobbs GJ. Alfentanil-mediated analgesia during propofol injection: no evidence for a peripheral action. $\mathrm{Br}$ J Anaesth. 1996;77(2):162-164. doi:10.1093/bja/77.2.162

2. Mangar D, Holak EJ. Tourniquet at $50 \mathrm{~mm} \mathrm{Hg}$ followed by intravenous lidocaine diminishes hand pain associated with propofol injection. Anesth Analg. 1992;74(2):250-252. doi:10.1213/ 00000539-199202000-00014

3. Picard P, Tramer MR. Prevention of pain on injection with propofol: a quantitative systematic review. Anesth Analg. 2000;90(4):963-969. doi:10.1097/00000539-200004000-00035

4. Singla B, Malde AD. A prospective observational study of injection pain in children with medium plus long chain triglyceride and long chain triglyceride propofol premixed with lignocaine. Indian J Anaesth. 2018;62(3):214-218. doi:10.4103/ija.IJA_506_17

5. Zirak N, Bameshki A, Yazdani M, et al. Lipid composition and lidocaine effect on immediate and delayed injection pain following propofol administration. Anesth Essays Res. 2016;10(1):29-32. doi:10.4103/0259-1162.164728

6. Sun J, Zhou R, Lin W, et al. Magnesium sulfate plus lidocaine reduces propofol injection pain: a double-blind, randomized study. Clin Ther. 2016;38(1):31-38. doi:10.1016/j.clinthera.2015.10.011

7. Jeong M, Yoon H. Comparison of the effects of lidocaine pre-administration and local warming of the intravenous access site on propofol injection pain: randomized, double-blind controlled trial. Int J Nurs Stud. 2016;61:209-218. doi:10.1016/j.ijnurstu.2016.06.012

8. Hong JM, Lee HJ, Cho AR, et al. Pretreatmet with 5\% lidocaine patch reduces cannula-induced and propofol-induced pain: a randomized, double-blind, placebo-controlled study. Korean $J$ Anesthesiol. 2016;69(5):468-473. doi:10.4097/kjae.2016.69.5.468

9. Euasobhon P, Dej-Arkom S, Siriussawakul A, et al. Lidocaine for reducing propofol-induced pain on induction of anaesthesia in adults. Cochrane Database Syst Rev. 2016;2:CD007874. doi:10.1002/ 14651858.CD007874.pub2

10. Madan HK, Singh R, Sodhi GS. Comparison of intravenous lignocaine, tramadol and ketorolac for attenuation of propofol injection pain. J Clin Diagn Res. 2016;10(7):UC05-08. doi:10.7860/JCDR/ 2016/20444.8118

11. Akbari H, Nasiri E, Nikkhah A, et al. Analgesic effects of ketamine, magnesium sulfate, and sodium-thiopental on propofol injection pain: a single-blind randomized clinical trial. Tanaffos. 2018;17(1):22-28.

12. Yu J, Zhang Y, Lu Y, Dong C. Preemptive dexmedetomidine to prevent propofol injection pain in children. Ir J Med Sci. 2015;184 (2):375-378. doi:10.1007/s11845-014-1122-3

13. Lee SH, Lee SE, Chung S, et al. Impact of time interval between remifentanil and propofol on propofol injection pain. J Clin Anesth 2016;34:510-515. doi:10.1016/j.jclinane.2016.06.029

14. Singh A, Sharma G, Gupta R, et al. Efficacy of tramadol and butorphanol pretreatment in reducing pain on propofol injection: a placebo-controlled randomized study. $J$ Anaesthesiol Clin Pharmacol. 2016;32(1):89-93. doi:10.4103/0970-9185.175703

15. Lee M, Kwon T, Kim S, et al. Comparative evaluation of the effect of remifentanil and 2 different doses of esmolol on pain during propofol injection: a double-blind, randomized clinical consort study. Medicine (Baltimore). 2017;96(10):e6288. doi:10.1097/MD.0000 000000006288
16. Kizilcik N, Menda F, Bilgen S, et al. Effects of a fentanyl-propofol mixture on propofol injection pain: a randomized clinical trial. Korean J Anesthesiol. 2015;68(6):556-560. doi:10.4097/ kjae.2015.68.6.556

17. Cheng D, Liu L, Hu Z. Prevention of anesthesia-induced injection pain of propofol in pediatric anesthesia. Pak J Med Sci. 2017;33 (3):752-756. doi:10.12669/pjms.333.12026

18. Sinha PK, Neema PK, Rathod RC. Effect of nitrous oxide in reducing pain of propofol injection in adult patients. Anaesth Intensive Care. 2005;33(2):235-238. doi:10.1177/0310057X0503300213

19. Kaabachi O, Chettaoui O, Ouezini R, et al. A ketamine-propofol admixture does not reduce the pain on injection compared with a lidocaine-propofol admixture. Paediatr Anaesth. 2007;17 (8):734-737. doi:10.1111/j.1460-9592.2007.02242.x

20. Davies AF, Vadodaria B, Hopwood B, et al. Efficacy of microfiltration in decreasing propofol-induced pain. Anaesthesia. 2002;57 (6):557-561. doi:10.1046/j.1365-2044.2002.02602.x

21. Wang J, Duan J, Xie C, et al. Comparison between intravenous nalbuphine and lidocaine in reducing propofol-induced injection pain during gastroscopy: a randomized controlled trial. Pain Ther. 2020;9(2):563-571. doi:10.1007/s40122-020-00188-y

22. Xing J, Liang L, Zhou S, et al. Intravenous lidocaine alleviates the pain of propofol injection by local anesthetic and central analgesic effects. Pain Med. 2018;19(3):598-607. doi:10.1093/pm/pnx070

23. Zhou J, Leonowens C, Ivaturi VD, et al. Population pharmacokinetic/ pharmacodynamic modeling for remimazolam in the induction and maintenance of general anesthesia in healthy subjects and in surgical subjects. J Clin Anesth. 2020;66:109899. doi:10.1016/j. jclinane.2020.109899

24. Wang F, Zhou Q, Shen M, et al. Efficacy and safety of remimazolam in procedural sedation and analgesia: a protocol for systematic review and meta analysis. Medicine (Baltimore). 2020;99(27):e20765. doi:10.1097/MD.0000000000020765

25. Sneyd JR, Rigby-Jones AE. Remimazolam for anaesthesia or sedation. Curr Opin Anaesthesiol. 2020;33(4):506-511. doi:10.1097/ACO.0000000000000877

26. Schuttler J, Eisenried A, Lerch M, et al. Pharmacokinetics and pharmacodynamics of remimazolam (CNS 7056) after continuous infusion in healthy male volunteers: Part I. Pharmacokinetics and clinical pharmacodynamics. Anesthesiology. 2020;132(4):636-651. doi:10.1097/ALN.0000000000003103

27. Schippers F, Pesic M, Saunders R, et al. Randomized crossover trial to compare abuse liability of intravenous remimazolam versus intravenous midazolam and placebo in recreational central nervous system depressant users. J Clin Pharmacol. 2020;60(9):1189-1197. doi: $10.1002 /$ jcph. 1614

28. Eisenried A, Schuttler J, Lerch M, et al. Pharmacokinetics and pharmacodynamics of remimazolam (CNS 7056) after continuous infusion in healthy male volunteers: part II. Pharmacodynamics of electroencephalogram effects. Anesthesiology. 2020;132(4):652-666. doi:10.1097/ALN.0000000000003102

29. Doi M, Morita K, Takeda J, et al. Efficacy and safety of remimazolam versus propofol for general anesthesia: a multicenter, single-blind, randomized, parallel-group, phase IIb/III trial. $J$ Anesth. 2020;34(4):543-553. doi:10.1007/s00540-020-02788-6

30. Bae JY, Choi DY, Woo CH, et al. The BIS and hemodynamic changes in major burn patients according to a slow infusion of propofol for induction. Korean J Anesthesiol. 2011;60(3):161-166. doi:10.4097/ kjae.2011.60.3.161

31. Kwak K, Kim J, Park S, et al. Reduction of pain on injection of propofol: combination of pretreatment of remifentanil and premixture of lidocaine with propofol. Eur J Anaesthesiol. 2007;24(9):746-750. doi: $10.1017 / \mathrm{S} 026502150600233 \mathrm{X}$

32. Salman AE, Salman MA, Saricaoglu F, et al. Pain on injection of propofol: a comparison of methylene blue and lidocaine. J Clin Anesth. 2011;23(4):270-274. doi:10.1016/j.jclinane.2010.09.008 
33. Angst MS, Mackey SC, Zupfer GH, et al. Reduction of propofol injection pain with a double lumen i.v. set. J Clin Anesth. 1997;9 (6):462-466. doi:10.1016/s0952-8180(97)00101-3

34. McCulloch MJ, Lees NW. Assessment and modification of pain on induction with propofol (Diprivan). Anaesthesia. 1985;40 (11):1117-1120. doi:10.1111/j.1365-2044.1985.tb10615.x

35. Scott RP, Saunders DA, Norman J. Propofol: clinical strategies for preventing the pain of injection. Anaesthesia. 1988;43(6):492-494. doi:10.1111/j.1365-2044.1988.tb06641.x

36. Turan A, Memis D, Kaya G, Karamanlioglu B. The prevention of pain from injection of propofol by dexmedetomidine and comparison with lidocaine. Can J Anaesth. 2005;52(5):548-549. doi:10.1007/ BF03016541

37. Coulthard P, Rood JP. An investigation of the effect of midazolam on the pain experience. Br J Oral Maxillofac Surg. 1992;30(4):248-251. doi:10.1016/0266-4356(92)90268-n
38. Chen X, Mou X, He Z, Zhu Y. The effect of midazolam on pain control after knee arthroscopy: a systematic review and meta-analysis. J Orthop Surg Res. 2017;12(1):179. doi:10.1186/ s13018-017-0682-0

39. Rouhi AJ, Zeraatchi A, Rahmani F, et al. Effect of oral midazolam in pain relief of patients need nasogastric tube insertion: a clinical trial study. J Res Pharm Pract. 2020;9(2):112-117. doi:10.4103/jrpp. JRPP_19_80

40. Xie $\bar{H}$, Lu F, Liu W, et al. Remimazolam alleviates neuropathic pain via regulating bradykinin receptor $\mathrm{B} 1$ and autophagy. J Pharm Pharmacol. 2021;rgab080. doi:10.1093/jpp/rgab080

\section{Publish your work in this journal}

Drug Design, Development and Therapy is an international, peerreviewed open-access journal that spans the spectrum of drug design and development through to clinical applications. Clinical outcomes, patient safety, and programs for the development and effective, safe, and sustained use of medicines are a feature of the journal, which has also been accepted for indexing on PubMed Central. The manuscript management system is completely online and includes a very quick and fair peer-review system, which is all easy to use. Visit http://www. dovepress.com/testimonials.php to read real quotes from published authors. 